recombinant IL-36 using isolated keratinocytes and bone marrow differentiated dendritic cells. We then assessed the sensitivity of IL-36R $\mathrm{R}^{\Delta \mathrm{K}}$ mice to IMQ-induced psoriasis, and compared it to mice presenting a complete IL-36R deficiency $\left(\mathrm{IL}-36 \mathrm{R}^{-/-}\right.$) and to their respective littermate controls (IL-36 $6^{+/+}$ and IL-36 $\left.\mathrm{R}^{\mathrm{fl} / \mathrm{fl}}\right)$. The severity of skin inflammation was assessed by ear thickness measured with a caliper. $\mathrm{H}$ and $\mathrm{E}$ staining was performed on treated and control ears. Total RNA was extracted from ears and mRNA levels of various cytokines were assessed by RT-qPCR.

Results IL-36 $\mathrm{R}^{-/-}$mice were strongly resistant to the induction of IMQ-induced psoriasis as assessed by ear thickness. IL-36 $\mathrm{R}^{\Delta \mathrm{K}}$ mice showed a similar macroscopic protection as IL-36 $\mathrm{R}^{-/-}$mice, demonstrating that IL-36 signaling in keratinocytes is critical in this model of psoriasis. Several proinflammatory genes upregulated by IMQ in IL-36 $\mathrm{R}^{+/+}$and IL-36 $\mathrm{R}^{\mathrm{fl} / \mathrm{fl}}$ mice were not stimulated in neither IL-36 $\mathrm{R}^{-/-}$nor in IL-36 $\mathrm{R}^{\Delta \mathrm{K}}$ mice. These genes included notably IL-17A or IL-22, both known to be crucial in psoriasis. Histological findings in IMQ-induced psoriasis include keratinocyte altered differentiation and hyperproliferation as well as inflammatory cell infiltration. Surprisingly and in contrast to IL-36R $\mathrm{R}^{-/}$mice, epidermidis thickness was not reduced in IL$36 \mathrm{R}^{\Delta \mathrm{K}}$ compared to IL-36 $\mathrm{R}^{\mathrm{fl} / \mathrm{fl}}$ control mice. This finding suggests that IL-36 signaling in keratinocytes does not induce keratinocyte hyper-proliferation but rather controls the development of downstream inflammatory responses in IMQ-treated ears.

Conclusions IL-36R signaling in keratinocytes is mandatory for the development of IMQ-induced psoriasis in mice. FACS studies are ongoing to characterize the inflammatory cell infiltrates in the different mouse lines.

Disclosure of Interest None declared.

\section{P094 EFFECTIVENESS AND SECURITY OF SECUKINUMAB IN PATIENTS WITH PSORIASTIC ARTHRITIS IN REAL CLINICAL PRACTICE}

${ }^{1} \mathrm{R}$ Hernández ${ }^{*},{ }^{1} \mathrm{~J}$ Uceda, ${ }^{2} \mathrm{~A}$ Perez Gil, ${ }^{1} \mathrm{JL}$ Marenco. ${ }^{1}$ Rheumatoloy; ${ }^{2}$ Dermatology, Valme University Hospital, Seville, Spain

\subsection{6/annrheumdis-2018-EWRR2019.83}

Career situation of first and presenting author Student for a master or a $\mathrm{PhD}$.

Introduction Recently new therapeutic targets have been approved for the treatment of psoriatic arthritis (PSA). We have limited experience in real clinical practice.

Objectives To assess efficacy, safety and tolerability of secukinumab in patients with active PsA.

Methods Descriptive, retrospective observational study on the efficacy and safety of secukinumab in patients who met CASPAR clasification criteria for PsA in follow-up in the Virgen de Valme hospital area. We evaluate, measures of disease activity by DAPSA in peripheral forms and ASDAS in axial forms. Statistical analysis The quantitative variables are expressed with means and standard deviations or medians and quartiles if the distributions are asymmetric, and the qualitative variables with percentages.

Results 12 patients were reviewed. 58.3\% were male.The average age of these patients is 47.67 years. The mean time of evolution in years of the disease was $8.33 .75 \%$ of the sample was not a smoker, and $83.3 \%$ of patients didn't consume alcohol excessively. 25\% were hypertensive. $8.3 \%$ had an associated dyslipidemia, and $16.7 \%$ had a symptomatic hyperuricemia.

Oligoarthritis form was the most frequent patterns of presentation with $46 \%$, followed by polyarthritis with $27 \%$. All patients had previously failed at least one DMARD, the most frequent being Methotrexate (41.7\%), Up to $75 \%$ of the patients were naïve to biological therapy. In patients refractory to biological therapy, the most commonly used drug in first choice was etanercept.

In peripheral presentation $80 \%$ of the patients had a moderate DAPSA at the beginning of the treatment and of these $62 \%$ passed to a low activity, while 38\% they remained with moderate activity.

In patients with high activity, up to $70 \%$ went to a low activity DAPSA, and in 30\% it decreased to moderate activity.

We have survival with the drug that has been 12.67 months with a DS of 7.07 months.

Treatment has been suspended in 3 patients, in one of them due to severe skin and joint breakout, in another due to nausea and headaches, and the last due to ineffectiveness.

Finally, regarding the safety data we have not had any severe adverse effects. $8.3 \%$ had mild adverse effects, the most frequent being infectious symptoms of the upper respiratory tract.

$8.3 \%$ had a primary failure (no response at any time) compared to $25 \%$ that has presented a secondary failure after a good initial response to treatment.

Conclusions Secukinumab provided sustained improvements in signs and symptoms in patients of active and was well tolerated, with a safety profile consistent with that reported previously.

\section{REFERENCE}

1. McInnes IB, Mease PJ, et al. Secukinumab, a human anti-interleukin-17A monoclonal antibody, in patients with psoriatic arthritis (FUTURE 2): Future 2 Study Group. Lancet 386(9999):1137-46.

Disclosure of Interest None declared.

\section{P095 INFLAMMATORY BOWEL DISEASE, DURING ANTI IL 17 TREATMENT}

${ }^{1} \mathrm{R}$ Hernández*, ${ }^{2} \mathrm{~A}$ Perez Gil, J Uceda, ${ }^{1} \mathrm{JL}$ Marenco de la Fuente. ${ }^{1} R$ heumatology: ${ }^{2}$ Dermatology, Valme University Hospital, Seville, Spain

\subsection{6/annrheumdis-2018-EWRR2019.84}

Career situation of first and presenting author Student for a master or a $\mathrm{PhD}$.

Introduction IL 17-A blocking with Secukinumab (SEK) has proved efficacy in the treatment of psoriasis and psoriasic artritis (PsoAr). Inflammatory bowel disease (IBD) is more common among patients with psoriasis and is cosidered as a part of the spectrum of spondyloarthritis. IL-17 is a cytokine required for the normal homesotasis of the bowel and its inhibition could initiate a subclinic disease.

Objectives We have observed 2 patients with IBD during therapy anti-IL17 for PsoAr.

Results Case 1 A 20-year-old woman with a diagnosis of plaque psoriasis since 12 years; she has been treated with topic treatment, phototherapy and Methotrexate. In November 2017 She presented skin worsening, (PASI 20, DLQI 20 and started treatment with SEK at a dose of $300 \mathrm{mg}$ every 4 